\title{
Genetics Selection Evolution reviewer acknowledgement 2014
}

Didier Boichard ${ }^{1}$, Jack Dekkers ${ }^{2}$, Helene Hayes ${ }^{1 *}$ and Julius van der Werf ${ }^{3}$

\section{Contributing reviewers}

The Genetics Selection Evolution editorial team would sincerely like to thank all of our reviewers who contributed to peer review for the journal in 2014.

Syuiti Abe

Japan

Mark Abney

United States of America

Hervé Acloque

France

Samuel E. Aggrey

United States of America

Andreia Amaral

Portugal

Peter Amer

New Zealand

Leif Andersson

Sweden

Jesus Arango

United States of America

Benoit Auvray

New Zealand

William Barendse

Australia

C. Simon Bawden

Australia

\author{
Bertrand Bed'Hom \\ France
}

Gary Bennett

United States of America

Jörn Bennewitz

Germany

\section{Camillo Berenos}

United Kingdom

Donagh Berry

Ireland

Jean-Pierre Bidanel

France

Piter Bijma

Netherlands

Marco Bink

Netherlands

Stephen Bishop

United Kingdom

Solomon Antwi Boison

Austria

Agnes Bonnet

France
Open Access
Er olution

Our reviewers who contributed to
Lanie Bourgeois
United States of America
Gudrun Brockmann
Germany
Fanny Calenge
France
Mario Calus
Netherlands
Rodolfo Juan Carlos Cantet
Argentina
Aurélien Capitan
France
Maria Carabaño
Spain
Roberto Carvalheiro
Brazil
Eduardo Casas
United States of America
Alessio Cecchinto

Open Access
Er

Our reviewers who contributed to
Lanie Bourgeois
United States of America
Gudrun Brockmann
Germany
Fanny Calenge
France
Mario Calus
Netherlands
Rodolfo Juan Carlos Cantet
Argentina
Aurélien Capitan
France
Maria Carabaño
Spain
Roberto Carvalheiro
Brazil
Eduardo Casas
United States of America
Alessio Cecchingto

Open Access
Er

Our reviewers who contributed to
Lanie Bourgeois
United States of America
Gudrun Brockmann
Germany
Fanny Calenge
France
Mario Calus
Netherlands
Rodolfo Juan Carlos Cantet
Argentina
Aurélien Capitan
France
Maria Carabaño
Spain
Roberto Carvalheiro
Brazil
Eduardo Casas
United States of America
Alessio Cecchingto

Open Access
Er olution

Our reviewers who contributed to
Lanie Bourgeois
United States of America
Gudrun Brockmann
Germany
Fanny Calenge
France
Mario Calus
Netherlands
Rodolfo Juan Carlos Cantet
Argentina
Aurélien Capitan
France
Maria Carabaño
Spain
Roberto Carvalheiro
Brazil
Eduardo Casas
United States of America
Alessio Cecchinto

Open Access
Er olution

Our reviewers who contributed to
Lanie Bourgeois
United States of America
Gudrun Brockmann
Germany
Fanny Calenge
France
Mario Calus
Netherlands
Rodolfo Juan Carlos Cantet
Argentina
Aurélien Capitan
France
Maria Carabaño
Spain
Roberto Carvalheiro
Brazil
Eduardo Casas
United States of America
Alessio Cecchinto

Open Access
Evolution

Lanie Bourgeois
United States of America
Gudrun Brockmann
Germany
Fanny Calenge
France
Mario Calus
Netherlands
Rodolfo Juan Carlos Cantet
Argentina
Aurélien Capitan
France
Maria Carabaño
Spain
Roberto Carvalheiro
Brazil
Eduardo Casas
United States of America
Aited to

Open Access
Er

Our reviewers who contributed to
Lanie Bourgeois
United States of America
Gudrun Brockmann
Germany
Fanny Calenge
France
Mario Calus
Netherlands
Rodolfo Juan Carlos Cantet
Argentina
Aurélien Capitan
France
Maria Carabaño
Spain
Roberto Carvalheiro
Brazil
Eduardo Casas
United States of America
Alessio Cecchingto

Open Access
Er

Our reviewers who contributed to
Lanie Bourgeois
United States of America
Gudrun Brockmann
Germany
Fanny Calenge
France
Mario Calus
Netherlands
Rodolfo Juan Carlos Cantet
Argentina
Aurélien Capitan
France
Maria Carabaño
Spain
Roberto Carvalheiro
Brazil
Eduardo Casas
United States of America
Alessio Cecchingto

Open Access
Er olution

Our reviewers who contributed to
Lanie Bourgeois
United States of America
Gudrun Brockmann
Germany
Fanny Calenge
France
Mario Calus
Netherlands
Rodolfo Juan Carlos Cantet
Argentina
Aurélien Capitan
France
Maria Carabaño
Spain
Roberto Carvalheiro
Brazil
Eduardo Casas
United States of America
Alessio Cecchinto

Open Access
Er

Our reviewers who contributed to
Lanie Bourgeois
United States of America
Gudrun Brockmann
Germany
Fanny Calenge
France
Mario Calus
Netherlands
Rodolfo Juan Carlos Cantet
Argentina
Aurélien Capitan
France
Maria Carabaño
Spain
Roberto Carvalheiro
Brazil
Eduardo Casas
United States of America
Alessio Cecchingto

Open Access
Er

Our reviewers who contributed to
Lanie Bourgeois
United States of America
Gudrun Brockmann
Germany
Fanny Calenge
France
Mario Calus
Netherlands
Rodolfo Juan Carlos Cantet
Argentina
Aurélien Capitan
France
Maria Carabaño
Spain
Roberto Carvalheiro
Brazil
Eduardo Casas
United States of America
Alessio Cecchingto

Open Access
Evolution

Lanie Bourgeois
United States of America
Gudrun Brockmann
Germany
Fanny Calenge
France
Mario Calus
Netherlands
Rodolfo Juan Carlos Cantet
Argentina
Aurélien Capitan
France
Maria Carabaño
Spain
Roberto Carvalheiro
Brazil
Eduardo Casas
United States of America
Aited to

Open Access
Evolution

Lanie Bourgeois
United States of America
Gudrun Brockmann
Germany
Fanny Calenge
France
Mario Calus
Netherlands
Rodolfo Juan Carlos Cantet
Argentina
Aurélien Capitan
France
Maria Carabaño
Spain
Roberto Carvalheiro
Brazil
Eduardo Casas
United States of America
Aited to

Open Access
Er

Our reviewers who contributed to
Lanie Bourgeois
United States of America
Gudrun Brockmann
Germany
Fanny Calenge
France
Mario Calus
Netherlands
Rodolfo Juan Carlos Cantet
Argentina
Aurélien Capitan
France
Maria Carabaño
Spain
Roberto Carvalheiro
Brazil
Eduardo Casas
United States of America
Alessio Cecchingto

Open Access
Er olution

Our reviewers who contributed to
Lanie Bourgeois
United States of America
Gudrun Brockmann
Germany
Fanny Calenge
France
Mario Calus
Netherlands
Rodolfo Juan Carlos Cantet
Argentina
Aurélien Capitan
France
Maria Carabaño
Spain
Roberto Carvalheiro
Brazil
Eduardo Casas
United States of America
Alessio Cecchinto

Open Access
Er

Our reviewers who contributed to
Lanie Bourgeois
United States of America
Gudrun Brockmann
Germany
Fanny Calenge
France
Mario Calus
Netherlands
Rodolfo Juan Carlos Cantet
Argentina
Aurélien Capitan
France
Maria Carabaño
Spain
Roberto Carvalheiro
Brazil
Eduardo Casas
United States of America
Alessio Cecchingto

Open Access
Er olution

Our reviewers who contributed to
Lanie Bourgeois
United States of America
Gudrun Brockmann
Germany
Fanny Calenge
France
Mario Calus
Netherlands
Rodolfo Juan Carlos Cantet
Argentina
Aurélien Capitan
France
Maria Carabaño
Spain
Roberto Carvalheiro
Brazil
Eduardo Casas
United States of America
Alessio Cecchinto

Open Access
Er

Our reviewers who contributed to
Lanie Bourgeois
United States of America
Gudrun Brockmann
Germany
Fanny Calenge
France
Mario Calus
Netherlands
Rodolfo Juan Carlos Cantet
Argentina
Aurélien Capitan
France
Maria Carabaño
Spain
Roberto Carvalheiro
Brazil
Eduardo Casas
United States of America
Alessio Cecchingto

Italy

Carole Charlier

Belgium

\footnotetext{
*Correspondence: helene.hayes@jouy.inra.fr

$\wedge$ Deceased

${ }^{1}$ INRA, UMR 1313 Génétique Animale et Biologie Intégrative, 78350

Jouy-en-Josas, France

2Department of Animal Science and Center for Integrated Animal Genomics,

lowa State University, Ames, IA, USA

${ }^{3}$ School of Environmental and Rural Science, University of New England,

Armidale, Australia
} 
Claude Chevalet

France

Ole F. Christensen

Denmark

\section{Christian Edel}

Germany

\section{Elena Ciani}

Italy

Samuel Adam Clark

Australia

Noelle Cockett

United States of America

John Cole

United States of America

Wouter Coppieters

Belgium

\section{Pascal Croiseau}

France

Ron Crump

United Kingdom

Ino Curik

Croatia

Yang Da

United States of America

Hans D. Daetwyler

Australia

Binyam Sime Dagnachew

Norway

Ingrid David

France

Dirk-Jan de Koning

Sweden

Gustavo de los Campos

United States of America

Jared Decker

United States of America

Julie Demars

France

Olivier Demeure

France

Leo Dempfle

Germany

Xiangdong Ding

China
Joerg Dodenhoff

Germany

Tom Druet

Belgium

\section{Susana Dunner}

Spain

Mathilde Dupont-Nivet

France

Klaus Ehrhardt

Germany

Jean Michel Elsen

France

Jeffrey Endelman

United States of America

Malena Erbe

Germany

Catherine Ernst

United States of America

Stéphane Fabre

France

Bin Fan

China

Pierre Faux

Belgium

\section{Majbritt Felleki}

Sweden

Jesús Fernández

Spain

Rohan Fernando

United States of America

Laurence Flori

France

Sylvain Foissac

United States of America

Luca Fontanesi

Italy

Alexis Fostier

France

Brad Freking

United States of America

Luis Alberto García Cortés Spain

Jose-Luis García-Marín

Spain
Dorian Garrick

United States of America

Giustino Gaspa

Italy

Mathieu Gautier

France

Helene Gilbert

France

Linus Girdland Flink

United Kingdom

Luis Gómez-Raya

Spain

Oscar González-Recio

Spain

Gregor Gorjanc

United Kingdom

Martien Groenen

Netherlands

Bernt Guldbrandtsen

Denmark

Satoshi Hamaguchi

Japan

Bevin Harris

New Zealand

Ben Hayes

Australia

Susanne Hermesch

Australia

Juan Manuel Herrero-Medrano

Netherlands

William Hill

United Kingdom

Jisca Huisman

United Kingdom

Noelia Ibañez

Spain

Eveline Ibeagha-Awemu

Canada

Ikhide Imumorin

United States of America

Jean-Luc Jannink

United States of America

Luc Janss

Denmark 
Steven Janssens

Belgium

John William Keele

United States of America

Kathryn Kemper

Australia

Brian Kinghorn

Australia

Brian Kirkpatrick

United States of America

Byung-Whi Kong

United States of America

Loeske Kruuk

Australia

Larry Kuehn

United States of America

Robert Lacy

United States of America

Laurence Lamothe

France

Catherine Larzul

France

Jan Lassen

United States of America

Tosso Leeb

Switzerland

Andres Legarra

France

Christina Lehermeier

Germany

Grégoire Leroy

France

Debby Lipschutz-Powell United Kingdom

\section{Zengting Liu}

Germany

Daniela Lourenco

United States of America

Tu Luan

Norway

Peipei Ma

China

Daniel Maizon

Argentina
Mahlako Makgahlela

South Africa

Christian Maltecca

United States of America

Zissis Mamuris

Greece

Esa Mantysaari

Finland

Christel Marie-Etancelin

France

Raluca Mateescu

United States of America

John McEwan

New Zealand

Ivica Medugorac

Germany

Gábor Mészáros

Austria

Theo Meuwissen

Norway

Ignacy Misztal

United States of America

Philippe Monget

France

Raphael Mrode

United Kingdom

Han Mulder

Netherlands

Sebastian Munilla

Argentina

Riccardo Negrini

Italy

Pablo Orozco-terWengel

United Kingdom

Mikhail Ozerov

Finland

Hubert Pausch

Germany

Francisco Penagaricano

United States of America

Paulino Pérez

Mexico

Miguel Pérez-Enciso

Spain
Florence Phocas

France

Jeffrey Plowman

New Zealand

Ricardo Pong-Wong

United Kingdom

Laercio R Porto-Neto

Australia

Jennie Pryce

Australia

Herman Raadsma

Australia

Sebastian Ramos-Onsins

Spain

Wendy Mercedes Rauw

Spain

Denis Réale

Canada

Kent Reed

United States of America

Norbert Reinsch

Germany

Carlo Renieri

Italy

Marcio Resende

United States of America

Anne Ricard

France

Silvia Teresa Rodriguez Ramilo

Spain

Andrés Rogberg-Muñoz

Argentina

Gary Rohrer

United States of America

Lars Rönnegård

Sweden

Sophie Rothammer

Germany

Suzanne Rowe

New Zealand

Goutam Sahana

Denmark

Juan Pablo Sanchez

Spain 
Magali San Cristobal

France

Anna Wensley Santure

United Kingdom

Honoo Satake

Japan

Larry Schaeffer

Canada

Karl Schmid

Germany

Dierck Segelke

Germany

Anna K. Sonesson

Norway

Tad Sonstegard

United States of America

Daniel Sorensen

Denmark

Juan Steibel

United States of America

Ismo Stranden

Finland

Guosheng Su

Denmark

Bolormaa Sunduimijid

Australia

Richard Tait

United States of America

Miika Jani Juhani Tapio

Finland
Frank Technow

United States of America

Robert Tempelman

United States of America

Jens Tetens

Germany

Elizabeth Thompson

United States of America

Bruce Tier

Australia

Miguel Angel Toro

Spain

Thierry Tribout

France

Daniel Vaiman

France

Julius van der Werf

Australia

Marc Vandeputte

France

Paul Vanraden

United States of America

Luis Varona

Spain

Roel Veerkamp

Netherlands

Johanna Vilkki

Finland

Beatriz Villanueva

Spain
Renaud Vitalis

France

Zulma Vitezica

France

Chunkao Wang

United States of America

Ji-Ping Wang

United States of America

Joel Ira Weller

Israel

Robin Wellmann

Germany

Pam Wiener

United Kingdom

Yvonne Wientjes

Netherlands

George Wiggans

United States of America

Klaus Wimmers

Germany

Anna Wolc

Poland

Kui Zhang

United States of America

Qin Zhang

China 